\title{
Nanotoxicology and nanoparticle safety in biomedical designs
}

This article was published in the following Dove Press journal:

International Journal of Nanomedicine

2 June 2011

Number of times this article has been viewed

\author{
Jafar $\mathrm{Ai}^{\mathrm{I}}$ \\ Esmaeil Biazar² \\ Mostafa Jafarpour ${ }^{3}$ \\ Mohamad Montazeri ${ }^{4}$ \\ Ali Majdi ${ }^{5}$ \\ Saba Aminifard ${ }^{5}$ \\ Mandana Zafari ${ }^{5}$ \\ Hanie R Akbari ${ }^{6}$ \\ Hadi Gh Rad ${ }^{7}$ \\ 'Department of Tissue Engineering. \\ Faculty of Advanced Technologies, \\ Tehran University of Medical \\ Sciences, Tehran; ${ }^{2}$ Department of \\ Chemistry, Islamic Azad University - \\ Tonekabon Branch, Mazandaran; \\ ${ }^{3}$ Department of Microbiology, Faculty \\ of Science, Islamic Azad University - \\ Tonekabon Branch, Mazandaran; \\ ${ }^{4}$ Faculty of Medical Sciences, Babol \\ University of Medical Sciences; \\ ${ }^{5}$ Young Researchers Club - Islamic \\ Azad University, Tonekabon Branch, \\ Mazandaran; ${ }^{6}$ Faculty of Medical \\ Sciences, Islamic Azad University - \\ North branch, Tehran; ${ }^{7}$ Faculty \\ of Medical Sciences, Islamic Azad \\ University - Tonekabon Branch, \\ Mazandaran, Iran
}

Correspondence: Esmaeil Biazar Islamic Azad University, Tonekabon Branch, Mazandaran, Iran

Tel +98 I92 4274415

Fax +98 I92427 44I I

Email e.biazar@tonekaboniau.ac.ir
Abstract: Nanotechnology has wide applications in many fields, especially in the biological sciences and medicine. Nanomaterials are applied as coating materials or in treatment and diagnosis. Nanoparticles such as titania, zirconia, silver, diamonds, iron oxides, carbon nanotubes, and biodegradable polymers have been studied in diagnosis and treatment. Many of these nanoparticles may have toxic effects on cells. Many factors such as size, inherent properties, and surface chemistry may cause nanoparticle toxicity. There are methods for improving the performance and reducing toxicity of nanoparticles in medical design, such as biocompatible coating materials or biodegradable/biocompatible nanoparticles. Most metal oxide nanoparticles show toxic effects, but no toxic effects have been observed with biocompatible coatings. Biodegradable nanoparticles are also used in the efficient design of medical materials, which will be reviewed in this article.

Keywords: nanotechnology, nanotoxicology, nanomaterials, nanobiomaterials

\section{Introduction}

The development of nanotechnology in different industries, its modernity, and also the lack of information on its negative effects on human health and the environment originate from the novel mechanisms that are also related to nanotoxicology. Some researchers are fundamentally against using nanomaterials in human medicine and in the environment while others are in favor. The important point here is that because there are many nanomaterials with many different uses, it is difficult to test all of them and estimate their effects on human health. Therefore, some scientists believe that their side effects are acceptable. ${ }^{1,2}$ Considering all factors, testing the effects of nanomaterials on mammals and the environment is necessary. Only with more research, and using scientific evidence, microscopy tools, and modern analysis methods, can we discover the advantages or disadvantages of their applications. New features of nano-sized materials can be found, including electrical conductivity, reactivity, stability, colorability, and toxicity. ${ }^{2}$ Carbon in the form of graphite is soft and malleable, although at a nano-sized scale, it becomes a nanocarbon tube, which is tougher than steel. One gram of catalyst with a diameter of $10 \mathrm{~nm}$ is about 100 times more reactive than a similar particle with a diameter of $1 \mu \mathrm{m}$. However, toxicity occurs with nano- and micron-sized particles. The important fact about nanoparticles is their remarkable reactivity, a characteristic that may result in toxicity effects. ${ }^{1,2}$ In this review article, nanobiomaterials used in the field of medical sciences are discussed, along with their toxicity effects. 


\section{Nanotoxicology}

Nanotoxicology is a branch of bionanoscience, which deals with the study and application of the toxicity of nanomaterials. Nanomaterials, even when made of inert elements such as gold, become highly active at nanometer dimensions. Nanotoxicological studies are used to determine whether and to what extent these properties may pose a threat to the environment and to human health. ${ }^{3}$

Nanoparticles play a remarkable role in toxicity, which is important for toxicologists, especially in respiratory diseases. Their size is an important factor in the occurrence of disease. Some studies on the different sizes of carbon and titanium oxide showed that reduction in nanoparticle size increases its toxicity in the lungs. Also notable is that combining some metals with each other causes complex toxicity, which does not occur with single metals. In 1975, a study showed the effect of oxidative stress caused by asbestos as the main factor in asbestosis and also in disturbing cell structure. In 1998, Zhang presented his findings on the effects of nanoparticles on respiratory toxicity and inflammation. ${ }^{4}$ Some of the particle features such as size, surface chemistry, and oxidative stress functions play important roles in nanotoxicity. Other features such as crystallinity, coating, and the longevity of particles have also been studied as important parameters. ${ }^{5}$ By gaining control over dangerous particles, we can increase the use of nanoparticles by reducing their harmful effects, and thus allowing them to be used in the curing of diseases. ${ }^{5-9}$

\section{Important factors}

\section{Size}

For particle toxicity, two factors are important: size and chemical compounds. A reduction in the size of nano-sized particles results in an increase in particle surface area. Therefore more chemical molecules may attach to this surface, which would enhance its reactivity and result in an increase in its toxic effects. ${ }^{8,9}$ Many studies on the absorption of nanoparticles from the mucus have examined these effects. After absorption, nanoparticles reach the blood stream and then spread through the tissue. In one study, $33 \%$ of $50 \mathrm{~nm}, 26 \%$ of $100 \mathrm{~nm}$, and $10 \%$ of $500 \mathrm{~nm}$ particles were discovered in mucosal and lymphatic tissues of the intestine. ${ }^{9}$ Nanoparticles larger than $1 \mu \mathrm{m}$ were weakly observed and nanoparticles larger than $3 \mu \mathrm{m}$ were occasionally seen in lymphatic tissues. Researchers have concluded that:

- Nanoparticles smaller than $100 \mathrm{~nm}$ are absorbed by the cells of the intestine but not the larger nanoparticles $(300 \mathrm{~nm})$.
- The absorption of smaller nanoparticles $(100 \mathrm{~nm})$ in the lymphatic tissue is greater than in intestinal cells.

- Intestinal cells cannot absorb nanoparticles larger than $400 \mathrm{~nm}$.

- Only nanoparticles smaller than $500 \mathrm{~nm}$ can enter the circulatory system.

Scientists are discussing the relationship between particle sizes and their penetration into mesenteric lymphatic glands, but so far have reached no agreement. ${ }^{9}$

In addition to being able to cross cell membranes, and reach the blood and various organs because of their small size, nanoparticles have a bigger surface to volume ratio than larger particles. Therefore more molecules of the chemical are present on the surface, which may be one of the reasons why nanoparticles are generally more toxic than larger particles of the same composition. ${ }^{9}$

\section{Particle surface}

In vitro studies have shown that very small particles have more pathological and destructive power on the lungs rather than the same particles of smaller size due to their larger surface area, greater tendency to conjugate, and energy sustainability. ${ }^{6-8,10-12}$

\section{Surface chemistry}

Geiser et $\mathrm{al}^{13}$ studied the interaction between particle surface chemistry and the lung's surface-lining layer. They found that, regardless of the nature of the surface, the particles will be submersed into the lining layer after deposition in the small airways and alveoli. This displacement is promoted by the surfactant film itself as its surface tension falls temporarily to relatively low values. ${ }^{13,14}$ On the other hand, the reactive groups on a particle surface will certainly modify the biological effects. For silica, it has been shown that surface modification of the quartz affects its cytotoxicity, inflammogenicity, and fibrogenicity. These differences are mainly due to particle surface characteristics. ${ }^{15}$ The specific cytotoxicity of silica is strongly correlated with the appearance of surface radicals and reactive oxygen species (ROS), which is considered a key event in the development of fibrosis and lung cancer caused by this compound. ${ }^{16}$ Although the type of particle does not seem to play an important role in whether it is embedded in the surfactant lining of the alveoli, the embedding process itself is crucial. Particlecell interaction is possible only after the immersion of the particulates in the lining fluid, and research is needed to study this phenomenon in detail in relation to the inhaled 
nanoparticles. Logically, as described in a report on silica, ${ }^{16}$ the reactive groups on nanoparticles influence their interaction with the lungs (or more generally with biological material). In some instances, it may be possible to predict the reactivity of the nanoparticle surface. The scarcity of data, however, suggests that verifying these predictions by laboratory testing would be sensible. The degree of hydrophobicity and hydrophilicity of a surface is the major feature used to estimate the toxicity. As well as size, it seems that the particle surface is critical in their absorption in the intestinal mucus. The absorption of nanoparticles produced by hydrophobic polymers is greater than that of nanoparticles produced by hydrophilic polymers. ${ }^{10-12}$

\section{Chemical components}

Chemical components of the particle surface have important effects on nanoparticles as they can react with metals. Iron can be affected by nanoparticles, which increases the induction of ROS in the free cell system. The surface modification of nanoparticles can reduce toxicity. Researchers have also shown that the toxicity of super paramagnetic iron oxide nanoparticles could be reduced by coating them with pullulan. ${ }^{10-12}$

\section{Dosage}

Toxicity and other responses depend on the prescribed dosage and substances used. Research has shown that a high dose of nanoparticles in small or big particles could be harmful to health. ${ }^{10-12}$

\section{Free radical production}

Most or all pathogenic particles produce free radicals in the free cell system and this ability causes oxidative stress, which gives rise to inflammation, cell destruction, and genotoxicity. The particle surface of free radicals can activate the redox cycle and cause particle toxicity. ${ }^{10,17}$

\section{Passage of nanomaterials through tissues}

In body engineering and design, there are three important sites in relation to the environment: skin, lungs, and the digestive tract. These organs protect the body from harmful environmental components. In other words, they are important organs in the transmission of nutrients, water, and oxygen. The skin acts as a barrier against the substances (apart from special elements such as oxygen for the retina and UV rays for vitamin D synthesis). ${ }^{18-21}$
Nano-sized particles can enter and penetrate some organs such as the lungs, intestine, and skin. Some can penetrate into the deepest layers of the skin (dermis). Their penetration depends on their size and nanoparticle surface features. It must be noted that in vitro tests must be carried out on nanoparticle toxicity before in vivo tests are performed. Figure 1 shows the areas of the body that should not be penetrated by nanoparticles.

The digestive (gastrointestinal) tract has a close relationship with the environment. Materials come into the body through the mouth and all nutrition is exchanged there, apart from gas. The histology of these three organs in relation to other places is different. The skin surface area of the body, which has an area of about $2 \mathrm{~m}^{2}$ and a thickness of about $10 \mu \mathrm{m}$, is composed of keratin cells. These cells form a barrier against transmitting ions. The amount of penetration is related to organ, age, and other agents. ${ }^{20,21}$

\section{Toxicity of nanoparticles}

Knowledge of the toxicity effects of these small substances is limited, but is rapidly growing. Many studies have shown that some nanoparticles demonstrate toxicity in biological systems. Thus research in the internal and external environment is needed; external studies can direct the internal studies. Some researchers have shown that most of the nanoparticles can release active oxygen and cause oxidative stress and inflammation by the RES (reticoendothelial system). Acute toxicity resulting from nanoparticles has been investigated in the mouths of rats. The results indicate that toxicity depends on the size, coating, and chemical component of the nanoparticles. Also, the systemic effects of nanoparticles have been shown in different organs and tissues. The effects on inflammatory and immunological systems may include oxidative stress or

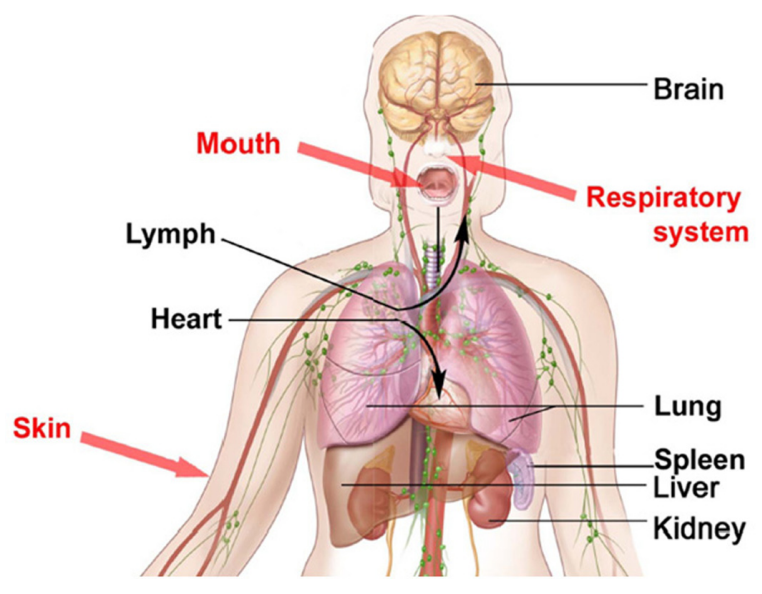

Figure I Parts of the body that should not be penetrated by nanoparticles. 
pre-inflammatory cytotoxin activity in the lungs, liver, heart, and brain. The effects on the circulatory system can include prethrombosis effects and paradox effects on heart function. Genotoxicity, carcinogenicity, and teratogenicity may occur as a result of the effects of nanoparticles. Some nanoparticles could pass the blood-brain barrier and cause brain toxicity (Figure 2); of course more studies are required. ${ }^{22-24}$

Due to the high loading of nanoparticles, macromolecule absorption will increase, so that they can cross through the digestive tract. Because, for example, lectin is such an immunologic material for coating, it can be toxigenic and also cause inflammatory responses or digestive stimulation. ${ }^{25,26}$

\section{Nanotitania toxicity}

Donaldson et al have shown that very small metal particles cause more inflammation than larger inhaled particles. Although the role of nanoparticles in toxicity is obscure, experimental evidence has shown that very small particles inhibit phagocytosis function more than larger particles. ${ }^{27}$ Researchers have investigated the effects of nanoparticles on microorganisms, invertebrates, and vertebrates. There is motivation to do more studies on microorganisms due to their importance in industry and medicine. For example, the antimicrobial effects of some particles have increased their use in various consumer goods such as clothes and medical equipment. After extensive investigation, $\mathrm{TiO}_{2}$ nanoparticles were introduced as antimicrobial particles and can be used as a coating material for medical equipment, because of their antimicrobial and mechanical properties. $\mathrm{TiO}_{2}$ can accumulate some types of oxygen, the same as hydroxyl radicals and hydrogen peroxide, which has been done by oxidation and restoration under light. The oxygen comes

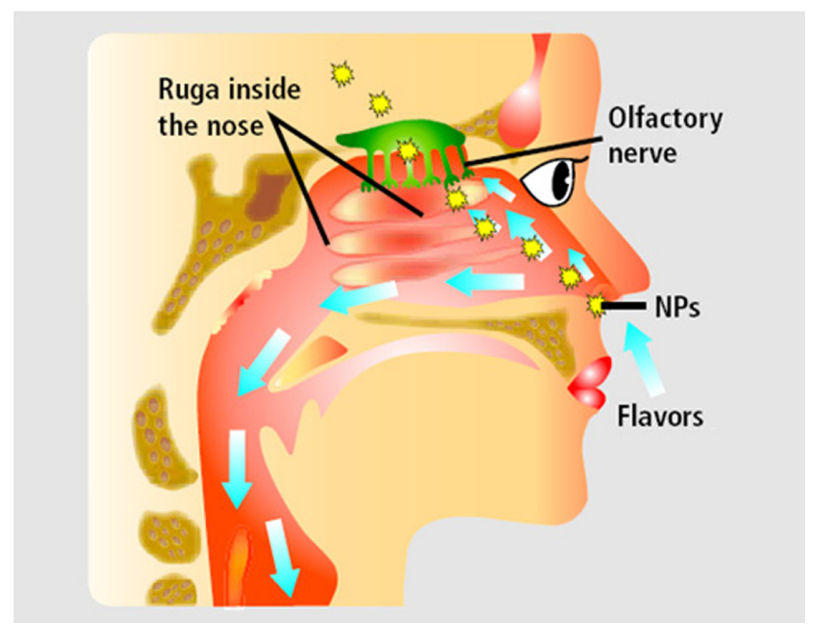

Figure 2 The passage of nanoparticles (NPs) from the nose to the cerebral system via the cibriform plate, which separates the nasal sinus from the brain and protects the nasal nerves and nervous receptors. into contact with UV rays, then a photo catalyst produces antimicrobial features which kill all bacteria with endotoxins which, in turn, have side effects in organisms. ${ }^{25-29}$ For example, in 2005, Lee et al discovered the killing features of $\mathrm{TiO}_{2}$ nanoparticles and carbon nanotubes on endospore bacteria, which were coated with multi-wall nanotubes. He found that combining UV radiation with $\mathrm{TiO}_{2}$ is more effective, although the combined $\mathrm{TiO}_{2}$ and nanotubes caused endospore concentration. Therefore, some spores survived because of this protection. ${ }^{30}$ The toxic effect of metal oxide particles on marine fibroblasts and marine monocytic macrophages have been investigated. The particles were added to cells and the comparative plating effect was assembled after 6 to 8 days, depending on cell type. The results showed that the toxicity of $\mathrm{ZrO}_{2}$ and $\mathrm{Al}_{2} \mathrm{O}_{3}$ particles $(\mathrm{d}=500-700 \mathrm{~nm})$ increases more than $\mathrm{TiO}_{2}$ particles $(130-180 \mathrm{~nm}) . \mathrm{Al}_{2} \mathrm{O}_{3}$ coated with $\mathrm{TiO}_{2}$ particles have shown the same toxic effects. Dendritic $\mathrm{TiO}_{2}$ has shown higher toxicity than other forms. The toxicity of ionic metals and other chemical materials differs among cells. The larger particles tend to show greater toxicity than the smaller particles. For example, the larger $\mathrm{TiO}_{2}$ particles cause a higher prevalence of the H-thymidine component than human monotypic macrophages. $\mathrm{ZnO}$ nanoparticles with a diameter of 500 to $3000 \mathrm{~nm}$ were placed on human fibroblasts for 24 hours. They were stained by hematoxin/eosin, which showed the extent of toxicity; the dead cells, which had been separated from the bottom of the glass, were not colored and the living cells still adhered and absorbed color. A digitizer was used to estimate the colored zone. The cells exposed to $\mathrm{Al}_{2} \mathrm{O}_{3}, \mathrm{TiO}_{2}, \mathrm{Fe}_{2} \mathrm{O}_{3}, \mathrm{Fe}_{3} \mathrm{O}_{4}, \mathrm{CO}_{2} \mathrm{O}_{3}, \mathrm{NiO}, \mathrm{Ga}_{2} \mathrm{O}_{3}, \mathrm{SnO}, \mathrm{SnO}_{2}$, and $\mathrm{HgO}$, showed no toxic effect. The results acquired from studies on $\mathrm{ZnO}, \mathrm{CuO}, \mathrm{Cu}_{2} \mathrm{O}, \mathrm{Cr}_{2} \mathrm{O}_{3}$, and $\mathrm{Ag}_{2} \mathrm{O}$ show that these particles have toxic effects. ${ }^{31}$

\section{Silver}

The antimicrobial effect of silver-coated surfaces has been studied with 16 types of bacteria. Silver nanoparticles are used in medical design, especially in dentistry. For example, nanosilver crystals are used in bandags as antimicrobial agents, but the use of silver nanoparticles depends on counteracting their positive (antimicrobial effect) and negative (cellular toxicity) effects. In one study, it was observed that nanosilver (12 nm) kills E scherichia coli. Figure 3 shows the effects of silver nanoparticles on bacteria by SEM analysis. ${ }^{32-35}$

\section{Zinc oxide $(\mathrm{ZnO})$ nanoparticles}

$\mathrm{ZnO}$ acts as an effective UV filter when used in suncreams and textiles. ${ }^{36}$ Some animal studies and autoradiography have 
shown that $\mathrm{ZnO}$ nanoparticles penetrate into the skin of rats and rabbits. Particles with a diameter of 50 to $100 \mathrm{~nm}$ can penetrate the skin because of the intracellular space of the corneum stratum, which is about $100 \mathrm{~nm}$ and the distance between the two layers is 0.5 to $1 \mathrm{~nm}$. During inhalation, the particles enter the deep zones of the lung where they are surrounded and excluded by macrophages before epithelial damage. The particles can attach to the epithelium (causing inflammation) and the entrance to the interstitium where they have chronic effects on cells and have the ability to move to the lymphatic nodes. ${ }^{37,38}$

\section{Fullerene toxicity}

$\mathrm{C}_{60}$ was first discovered by Korto in 1985, who said that $\mathrm{C}_{60}$ has 20 dimensions in different situations and is composed of apexes and 20 faces. ${ }^{39}$ Some features of $\mathrm{C}_{60}$ show that it has potential for use. For example, the first use of $\mathrm{C}_{60}$ has been in optics and conductors. Also, it is used to produce various sanitary products such as creams. Research has proved the antioxidative properties of $\mathrm{C}_{60}{ }^{40}$ For instance, Wang suggested in 1999 that some $\mathrm{C}_{60}$ parasynthetics dissolved in water can inhibit lipid oxidation and radicalization and super oxidation. ${ }^{41}$ The risks of $\mathrm{C}_{60}$ particles were detected for every three groups of cells if the density was more than $50 \mathrm{ppb}$. The $\mathrm{C}_{60}$ particles affect the cellular membrane due to the induced peroxyl free radicals. Conversely, L-ascorbic acid inhibits risk and membrane damage. Some ecotoxicology research has shown that $\mathrm{THF}$ is a suitable solution for $\mathrm{C}_{60}$ spreading, but the important point is that THF may change toxicity risk in particles, although more information is required. ${ }^{42}$ In 1998, Kamat et al described lipid oxidativity by $\mathrm{C}_{60}$ using the microorganisms of the liver. The study showed that changed fullerene can be toxic, but this toxicity depends on its group factors, which is not a feature of fullerene. ${ }^{43}$ Fullerenes can be used in drug delivery systems. The preparation methods for fullerene solution are very important. To prepare this solution, fullerene must first be dissolved in polar solvent, which is able to be dissolved
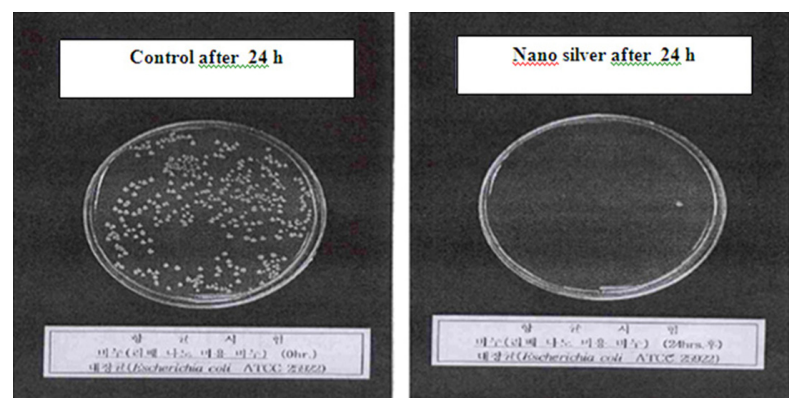

Figure 3 Antibacterial effect (Escherichia coli) of silver nanoparticles and silver particles (control) over 24 hours. in water. For example, scientists use tetrahydrofuran. The quick separation of organic solvent from aqueous fullerene solution is impossible. In fact, watery solution causes toxicity. In one new study, the researchers investigated the effects of these materials on fish, and described the fullerene antioxidative effect. Cerebral damage could occur due to respiratory medicines such as D-ethyl ether. Tetrahydrofuran has an ether-like effect and is very toxic. In fact, tetrahydrofuran caused cerebral damage, but fullerene had no such effects. ${ }^{44}$

\section{Carbon nanotubes}

Carbon nanotubes have a seamless graphite cylinder, which has featured in a number of studies, especially in medical science, it is also interesting to study because of its paradoxical effect on the body. Nanotubes can be made impure by other metals such as iron and initiate the redox cycle and ROS gathering (the same as asbestosis). ${ }^{45,46}$ Carbon nanotubes may have numerous or single walls. Other structures are nanowires, nanorods, and nanosprings. We consider that the nanoparticles can be compressed, but nanotubes such as nanoropes, which are special nanotubes, can rotate around each other and make a larger fiber. Carbon nanotubes tend to twist in the form of a rope, which can be a problem (especially in the lungs). ${ }^{49}$ Nanotubes are structures that may behave as nanoparticles or fibers. ${ }^{41}$ For example, lung toxicity will occur at high doses of singleor multiple-wall carbons, but if their amount and dose are low, inflammation will occur in the lungs. Results have shown that carbon nanotubes at high dose are toxic for organisms, and accordingly, health scientists have defined them as dangerous and suggested manipulation of the nanoparticles. ${ }^{47}$ Also, an increase in the functional degree of single-wall carbon nanotubes displays lower toxicity than multi-wall nanotubes in in vitro tests on fibroblasts. Naturally, carbon nanotubes have an unchangeable and inactive surface. The length of time needed for the detection of toxicity in carbon nanotubes is still under study. Carbon nanotubes with a single wall are shorter and, if multi-walled, are about $100 \mathrm{~nm}$ long. In this context, we can mention the asbestosis because the length of those nanofibers are similar. In fact, some of the carbon nanotubes seem to be changeable and have the ability to rotate. Nanotubes can have fibrogenic effects such as big granulomas, which have been tested in rodents. Pleural mesothelioma is a cancer caused by asbestos exposure, yet rarely occurs in animals. Although these experiments are slow, nanotubes can be changed into larger particles by rotation, which gives us abnormal results. ${ }^{48-51}$ One way to reduce the toxicity of carbon 
nanotubes is coating or functionalization. Functionalization can affect the properties of the carbon nanotubes, especially their toxicity. ${ }^{52}$

\section{Nanodiamond particles}

Biological contacts may be tolerated well by the body, but small, divided particles of these materials may cause carcinogenic effects. ${ }^{53}$ The variation in size of the particles influences the histological reaction and cytosine production. ${ }^{53,54}$

Diamond dust does not stimulate the active oxygen metabolite by polymorphonuclear (PMN) leukocytes (a clear path to chronic inflammation and lung tissue damage).

Twenty-one percent of PMN cells of 3- $\mu \mathrm{m}$ diamond-dust crystals at a viscosity of $2 \mathrm{mg} / \mathrm{cm}^{3}$ were phagocytized after 45 minutes, but did not cause any chemotactic activity. ${ }^{55}$ In this study, ${ }^{55}$ horse and pig neutrophils were exposed to urea, apatite hydroxyl, and pyrophosphate. The results demonstrated that they induced superoxide and peroxide at the same viscosity and temperature, but exposing the neutrophils to the diamond crystal did not cause this effect at $37^{\circ} \mathrm{C}$. The hydroxyapatite crystals stimulate some enzymatic reactions and the production of crude mitochondrial fractions (CMF). Hydroxyapatite (HA) particles usually have scaly surfaces with negative surface load (or zeta potential), but diamond particles are flat with low surface load or without load. Diamond is accepted in the scientific community as a biomaterial in the 21 st century and is used in coatings for synthetic heart valves, orthopathy designs, joint substitutes, catheters, stent ortheopathic pins, and tooth roots. Figure 4 shows diamond nanoparticle coating on a hip implant, which will increase strength, but the release of nanoparticles as a result of scratching or abrasion can cause problems for the body. In vitro experiments with orthopathic pain coated by diamond-like carbon (DLC) implanted in sheep showed the low bioactivity of diamonds. Subconium implants coated with DLC in mice tibia for 30 days showed good osteogenesis in relation to the tissue implant. It is suggested

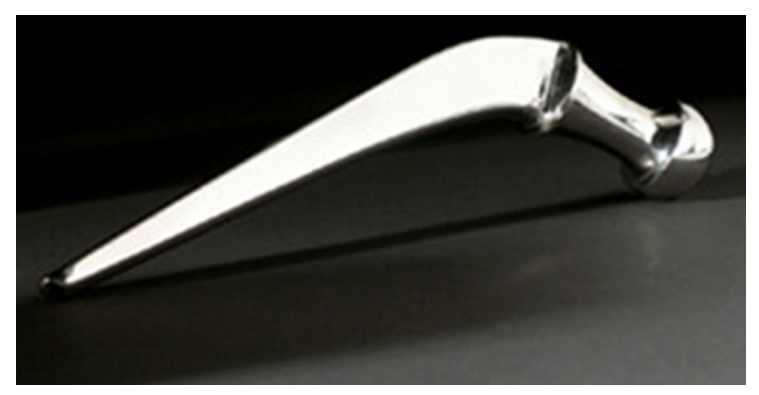

Figure 4 The implants with covers as nano diamond particles show better resistance and consistency. that diamond coatings (during the chemical vaporization sedimentation process) used in artificial joints, stimulate the lower immune system (immune reactivity) and in vitro tests have shown that diamond abraded the releasing particles. The toxicity study of DLC coated nanoparticles on vicinity rust-proof steel implants in the hip shows good cell adhesion (human fibroblasts) and bioadjustment, and in vivo tests of rust-proof steel covered with DLC with $4 \mathrm{~mm}$-cylinders implanted into cortical bone and sheep myometrical tissue did not appear to cause any problems. Chrome-cobalt cylinders coated with DLC were implanted in the muscle of rats: all implants showed good adjustment 90 days after the tests. Therefore, DLC covers are bioadjusted in both in vitro and in vivo environments of skeletal myometrical systems. Other DLC covers for osteoplants also show good bioadjustment. In most cases, erosion on Amorph diamond in comparison with commonly used material in the hip joint is immaterial. The carbon and diamond particles cultured by macrophages are digested without harmful effects. For example, the cells, which were exposed to a large amount of diamond dust, show recovery in less than 30 hours. The small particles of HA, silicon carbide, polymethylmethacrylate, and flouted diamonds in culture media, were compared with human serum monocytes at $0.5 \mathrm{mg} / \mathrm{cm}^{3}$ viscosity. The monocyte morphology changed after silicon carbide and HA digestion, but no differences were seen after diamond absorption. $^{55}$

\section{Magnetic nanoparticles}

Iron oxide nanoparticles are applied in biological detection and treatment. ${ }^{56,57}$ Magnetic nanoparticles have been used in photogene, targeting drug delivery, cell separation, cancer therapy, imaging, and magnetic hyperthermia for cancer therapy, and also for tissue engineering. Bioadjusted super paramagnetic nanoparticles, 2 to $30 \mathrm{~nm}$, with citric acid or methyl carboxyl dextrin were tested on rats and showed that these nanoparticles cause diarrhea and may lead to animal death while citrate itself does not cause toxicity. ${ }^{56-58}$ Of course, it is not clear whether the harmful agent was manganese, iron, or neither of these. The effects of bioadjusted coating layers have been studied on magnetic nanoparticles. Bovine serum albumin (BSA), poly-L-lactic acid (PLLA), and/or poly- $\varepsilon$-caprolactone were coated with eight nanometric particles. The toxicity reduction in human fibroblast cells was reported with super paramagnetic particles coated with pullulan. Uncoated iron oxide particles of $20 \mathrm{~nm}$ cause toxicity in human skin fibroblasts. Iron oxide nanoparticles of $9 \mathrm{~nm}$ were 
coated with polyvinyl alcohols (PVA), which have thiol, amine, and carboxylic acid functional groups. The results show that PVA nanoparticles, when combined with thiol, carboxyl, and PVA, poison melanoma cells. Higher levels of toxicity were seen in PVA nanoparticles combined with amine groups, especially when they are at high viscosity. Therefore, amino groups increase absorption into cells. Also, iron oxide nanoparticles coated with $9 \mathrm{~nm}$ citrate were studied on rat macrophages. NMR studies and atomic absorption have shown that the increased absorption of nanoparticles can easily be seen by a confocal microscope (iron oxide particles were marked with green rudimin and were exposed to the cells for 90 minutes at $37^{\circ} \mathrm{C}$ ). After 1 day, the adhesive cells could be distinguished by their fluorescent color, which is the standard color for these studies (extracellular membrane structures). After 45 minutes of overlapping, the cells were washed and studied with a confocal microscope. With 488-nm optic aggravation, both green rudimin suffusions and red ANEPPS suffusions were monitored simultaneously. The results show that the iron oxide nanoparticles were absorbed by the cells. The high level of fluorescence of ANEPPS in the same zone causes this orientation, that is, iron oxide nanoparticles are concentrated in coated membrane vesicles. The cell lyses occur after exposing them for different lengths of time. During the short overlapping times, there was a distinctive increase in cell oxidation and MDA. Therefore, oxidative stress is temporary and the cells keep their functionality and could be useful for magnetic uses. We can see the relevance of another type of nanoparticle for the body's immune system in super paramagnetic heating for terminating carcinomatous cells using iron oxide magnetic particles and their composites. The systemic temperatures can be created by a low dose of iron oxide. In clinical use, the iron oxides are absorbed by the reticuloendothelial system. Even if the iron particles surround the tumor environment, certain systemic risks can harm the reticuloendothelial system by heating the particle. The amount and effect of heating resulting from the iron oxide nanoparticles differ. We can see peculiar magnetic features and greater improvement because of the magnetic cross section, which depends on the component conjugated with nanoparticles. Nanoparticles activate the complementary system, which is a normal response of the immune system to external materials. In this system, the absorption of plasma protein on the surface of nanoparticles causes their detection, clearance, and digestion by RES system macrophages. ${ }^{59}$

\section{Nanomaterial toxicity in drug delivery systems}

Nanostructures can be used to transmit drug targets (as a drug or transmitter) or increase drug effectiveness. ${ }^{60}$ Nanotechnology is important, especially for detecting and treating cancer, although many problems have yet to be solved. Essentially, nanostructures are studied for gene transmission and their usage in vaccination and cancer treatment. Gene transmitting has been done both in vitro and in vivo with different types of nanoparticles. In nanoparticle distribution in the body, immunological, pathological, pharmacological, and pharmacodynamic factors (time-base level of absorption, metabolism, and drug clearance) control the distribution of biological behaviors of nanoparticles in the body. In an in vivo environment, the fate of nanoparticles depends on these factors. After intravenous effusion of nanoparticles on rats, nanoparticles were rapidly removed from the blood. This action was done by the immune and reticuloendothelial system without considering the particle features. Autoradiographic studies have shown that nanoparticles are usually concentrated in the liver and bone marrow. Cyanoacrylic polymeric nanoballs decrease gradually in the liver and are cleared from the body in the feces and urine. ${ }^{61-63}$ Nanoball clearance was completed after 7 days. Briefly, we can say that the liver is an important organ for the clearance of nanoparticles. The highest concentration of nanoparticles is found in liver cells. On the other hand, the concentration of these particles on mononuclear phagocytes causes the drug to keep away from the target cells. We suggest several methods for preventing this event. One is magnetic directional guidance of intravenous particles out of the body. It seems that this effect is intensified by increasing tumor angiogenesis. For example, albumin microballs with magnetic characteristics containing doxorubicin can penetrate into tumors more effectively than free drugs. On the other hand, by using nanoparticles to introduce the drugs into the body, drug distribution coincides with particle distribution. Changing the distribution model for nanoparticles is useful for some diseases such as cancer and also for reduction in drug toxicity. This advice has been used for anticarcinoma drugs and doxorubicin. The use of this drug has been limited because of chronic and acute cardiac failure. Doxorubicin nanoballs have lower toxicity than free drugs. Using this method, it is possible to transmit drugs into the MPS system with mononuclear phagocytes. Researchers have detected that ampicillin density in the liver is 20 times more than when free drugs 
are used. Intramuscular injection of labeled polymethyl methacrylate nanoparticles showed that these particles were fully absorbed after 70 days. After subcutaneous injection, the concentration of the nanoparticles decrease slowly in the tissues. Moreover, the injection of nanoparticles causes better performance in drug delivery systems, which results in increased bioavailability of peptic drugs after the drug injection has metabolized freely. As research has shown, nanoparticles are picked up on a large scale by mononuclear phagocytes after intravenous injection. On the other hand, it has been proved that distribution in the body can affect nanoparticle toxicity. Thus, in a new drug transmission system based on polymers, we must consider the possibility of activation or inhibition of mononuclear phagocytes. In this case, we must pay attention to changes in blood viscosity because the clot could be composed of nanoparticles and could cause red blood cell destruction. For other prescription methods, such as from the skin or through oral means, the stimulation of the local tissue is important. ${ }^{64-66}$

One of the problems of using nanoparticles in pharmacology is their uptake by the mononuclear phagocytosis system as they exist in the liver and spleen, although the targeting of the liver by nanoparticles may be suitable during treatment of liver diseases such as turmeric metastasis or hepatitis. Oligonucleotides can be used to control gene expression when they migrate to the liver when bonded with biodestructible polyalkyl cyanoacrylate nanoparticles. Besides the reduction in treatment effect, the uptake of nanoparticles in the liver may have a negative effect on liver function. The inflammatory responses by glycoprotein acid diffusion were caused by hepatocytes. ${ }^{67}$

Although nanoformulations for nanotransmission is increasingly proposed without any reduction in drug activity, in one study, insulin-cytosine nanoparticles were compared with cytosine solution and cytosine powder formulation. Insulin-cytosine nanoparticles showed a decreasing effect in viability and blood sugar in both mouse and sheep models. In pharmacology, the organs or cells are equally important, but nanoparticle effects on cells are important. For encapsulated drug activity, releasing of intracellular fluid is required; however, cell absorption for nanoparticles that are $20 \mathrm{~nm}$ or less in size do not need the endocytic mechanism. Chemical features such as surface load may affect intracellular nanoparticles. PLGA nanoparticles are digested by cells during endocytosis. One of the effects of nanoparticle formulation is an increase in cell encapsulation. Use of nanoparticles as drug transmitters may reduce combined drug toxicity. Usually, drug toxicity profiles are studied extensively while nanoparticle results are not described. Therefore, we cannot differentiate between nanoparticle and drug toxicity. In vitro toxicity is decreased (when compared with free indometacin) for nanospheres loaded with indometacin (at sizes smaller than $200 \mathrm{~nm}$ ) combined with polymethoxy polymers (ethylene glycol/poly[E-caprolacton]). However, toxicity in nanopolymers are yet to be profiled. Based on in vitro acute toxicity studies, $\mathrm{LD}_{50}$ was $1.47 \mathrm{~g} / \mathrm{kg}$, and $50 \%$ of $\mathrm{LD}_{50}$ tested for 7 days did not induce toxicity in the heart, lung, liver, or kidney, whereas it was detected 8 days after treatment and usage. Quantum dots of 15-20 nm show that these particles migrate and concentrate in lymphatic nodes around the injection zone. Nanoparticles can be injected in veins in a colloid drug releasing system. They can also be injected in the muscle or used in oral or optical applications. With intravenous injections, quantum dots move to the reticuloendothelial system (RES), which releases them into the liver, spleen, brain, bone, and also cardiac, renal, and respiratory systems. This distribution changes when the hydrophobicity and the surface load are corrected with the nanoparticle covers on different surfaces (same as surface correction with PEG and poloxamer). The nanoparticles are largely removed by mononuclear phagocytes after intravenous injection. On the other hand, it has been proved that the nano distribution model in the body can affect its toxicity. In fact, changing the drug distribution and pharmacokinetics could help develop a new model for drug effect and metabolism. ${ }^{67}$ For example, doxorubicin cardiac and bone toxicity may be increased after combing this drug with smaller nanoparticles. Accordingly, in a novel drug transmission system based on polymers, we must consider the possibility of mononuclear phagocyte activation or inhibition. For example, with changing nanosuspension surface features, their effects can be improved even more. Nanoparticle adhesion is an important factor in bioapplication, drug absorption, and reduction of drug clearance. Therefore, using mucus on nanoparticle surfaces can improve drug absorption..$^{67,68}$

\section{Nanomaterial toxicity mechanism for drug delivery in cancer therapy}

As mentioned before, one of the advantages of using nanoparticles in drug formulations is their potential for crossing the blood-brain barrier, although this function could have harmful effects. The nanoparticles have a toxic effect on cerebral endothelium cells. Of course, this is not 


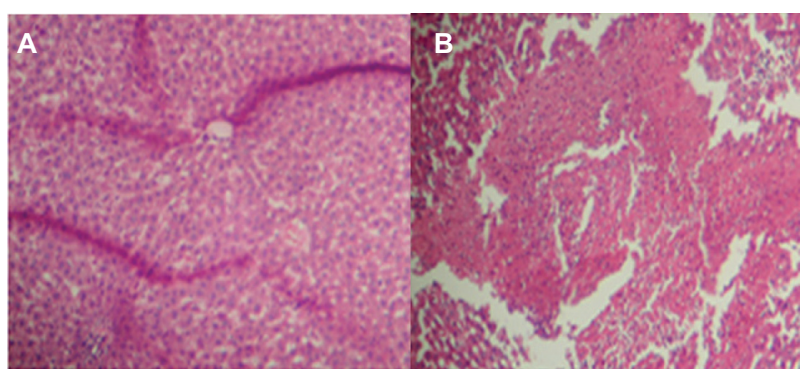

Figure 5 Liver tissue damage due to injection of acetaminophen at two different concentrations. A) shows little damage due to acetaminophen nanodrug injection and $\mathbf{B})$ shows normal drug injection. ${ }^{69}$

true when applied to all nanoparticles of the same size. The physical features of biological materials and their ability to adhere to nanoparticles are important. When nanoparticles with different surface features are tested, neutral nanoparticles and anionic nanoparticles do not have any effect on the blood cerebral system, while a high density of cationic nanoparticles has toxic effects on the blood-brain barrier system. The surface load of nanoparticles must be considered with regard to their toxicity effect and cerebral distribution profiles. The nanoparticles cause material transmission into the brain via the polysorbate surfactant Tween. The transmission mechanism by endocytosis of lipoprotein receptors at low density of endothelial cells after the absorption of blood plasma lipoprotein with nanoparticles is suggested..$^{70,71}$

The effect of paclitaxel entrapment and toxic drug effects in polysorbate/steel alcohol nanoparticles was studied in rats in a cerebral injection model. The results show that paclitaxel entrapment in nanoparticles clearly increases cerebral drug absorption and its toxicity in tumor cell P-glycoprotein expression. ${ }^{72}$

After oral injection, only $10 \%$ of $60 \mathrm{~nm}$ polystyrene particles were recovered again from the digestive tract. Most of these particles were found in the lymphatic tissue, such as Peyer's patches and the lymphatic tissues in the colon. The injection of nanoparticles in the dermis is optimal for cationic particles in the size range of 50 to $500 \mathrm{~nm}$, and less effective with anionic and neutral particles of any size. Migration to the draining lymphatic nodes is important for detection and treatment. Polyisobutyl cyanoacrylate nanoparticle formulation and fluorescent quantum dots are found entrapped in the draining lymphatic nodes. ${ }^{72}$

The structures and features of nanogold particles make them useful for estimating their biological use. Although some of the effects were seen by using these systems at high density, using $2 \mathrm{~nm}$ gold cationic particles in microbiological estimates and in vitro hemolysis show slight toxicity. Since the anionic particles are comparatively nontoxic, these very small $2 \mathrm{~nm}$ gold nanoparticles are nontoxic when they are used in rats for tumor treatment. ${ }^{73-75}$ If we compare free TNF with PEG colloidal nanoparticles conjugated with TNF we observe increasingly antitumoral activity. While the best isomer inhibitors of topoisomerase are formulated in nanoparticle lipid, its antitumoral activity will increase in the in vivo model of human tumors grown on rat glands. ${ }^{76}$

\section{Conclusion}

In this review article, nanobiomaterials, which are used in the field of medical science, have been discussed and their toxicity effects investigated. It is obvious that most nondegradable nanoparticles considered in this review are toxic and can influence the body's cells. The biocompatible coatings improve the performance of these nanoparticles, reduce their toxicity, and do not result in negative effects on cells. The emphasis of this study is to use biodegradable and biocompatible nanobiomaterials. Some synthetic materials such as PLGA, PCL, or natural materials such as collagen or chitosan can themselves be used as nanoparticles in medical applications.

\section{Disclosure}

The authors declare no conflicts of interest in this work.

\section{References}

1. Colvin VL. The potential environmental impact of engineered nanomaterials. Nat Biotechnol. 2003;21:1166-1170.

2. Borm PJA. Particle toxicology: from coal mining to nanotechnology. Inhal Toxicol. 2002;14:311-324.

3. Buzea C, Pacheco I, Robbie K. Nanomaterials and nanoparticles: sources and toxicity. Bio Interphases. 2007;2:MR17-MR71.

4. Zhang QW, Kusaka Y, Sato K, Nakakuki K, Kohyama N, Donaldson K Differences in the extent of inflammation caused by intratracheal exposure to three ultrafine metals: role of free radicals. J Toxicol Env Heal. 1998;53:423-438.

5. Schlesinger RB. Toxicological evidence for health effects from inhaled particulate pollution: does it support the human experience? Inhal Toxicol. 1995; 7:99-109.

6. Oberdorster G, Ferin J, Lehnert BE. Correlation between particle-size, in-vivo particle persistence, and lung injury. Environ Health Persp. 1994;102:173-179.

7. Holsapple MP, Farland WH, Landry TD, et al. Research strategies for safety evaluation of nanomaterials, part II: Toxicological and safety evaluation of nanomaterials, current challenges and data needs. Toxicol Sci. 2005;88:12-17.

8. Linkov I, Satterstrom FK, Corey LM. Nanotoxicology and nanomedicine: making hard decisions. Nanomedicine: Nanotech Bio and Med. 2008;4:167-171.

9. Hyuk Suh W, Suslick SK, Stucky Galen D, SuhYoo-Hun. Nanotechnology, nanotoxicology, and neuroscience. Pro Neurobiol. 2009; 87(3):133-170. 
10. Singh S, Shi T, Duffin R, et al. Endocytosis, oxidative stress and IL-8 expression in human lung epithelial cells upon treatment with fine and ultrafine $\mathrm{TiO}_{2}$ : role of the specific surface area and of surface methylation of the particles. Toxico Appl Pharmacol. 2007;222(2): 141-151.

11. Clift Martin JD, Rutishauser BR, Brown DM, et al. The impact of different nanoparticle surface chemistry and size on uptake and toxicity in a murine macrophage cell line. Toxicol-Appl Pharmacol. 2008;232: 418-427.

12. Oberdo G. Safety assessment for nanotechnology and nanomedicine: concepts of nanotoxicology. J Inte Med. 2010;267:89-105.

13. Geiser M, Schurch S, Gehr P. Influence of surface chemistry and topography of particles on their immersion into the lung's surface-lining layer. J Appl Physiol. 2003;94:1793-1801.

14. Gehr P, Geiser M, Hof VI, Schurch S. Surfactant ultrafine particle interactions: what we can learn from PM10 studies. Phil Trans $R$ Soc Lond A. 2000;358:2707-2718.

15. Schins RP, Duffin R, Hohr D, et al. Surface modification of quartz inhibits toxicity, particle uptake, and oxidative DNA damage in human lung epithelial cells. Chem Res Toxicol. 2002;15:1166-1173.

16. Fubini B. Surface reactivity in the pathogenic response to particulates. Environ Health Perspect. 1997;105:1013-1020.

17. Hussain S, Boland S, Baeza-Squiban A, et al. Oxidative stress and proinflammatory effects of carbon black and titanium dioxide nanoparticles: role of particle surface area and internalized amount. Toxicol. 2009;260:142-149.

18. Buzea C, Pacheco Blandino Ivan I, Robbie K. Nanomaterials and nanoparticles: sources and toxicity. Biointerphases. 2007;2:7-172.

19. Geiser M, Kreyling WG. Deposition and biokinetics of inhaled nanoparticles. Part Fibre Toxicol. 2010;7:2-6.

20. Medina C, Santos-Martinez MJ, Radomski A. Nanoparticles: pharmacological and toxicological significance. British J Pharmacol. 2007;150:552-558.

21. Revell PA. The biological effects of nanoparticles. Nanotech Perceptions. 2006;2:283-298.

22. Mühlfeld C, Gehr P, Rothen-Rutishauser B. Translocation and cellular entering mechanisms of nanoparticles in the respiratory tract. Swiss Med Wkly. 2008;138:387-391.

23. Rutishauser R, Mühlfeld C, Blank F, Musso C, Gehr P. Translocation of particles and inflammatory responses after ex-posure to ine particles and nanoparticles in an epithelial air-way model. Part Fibre Toxicol. 2007:4:9-15.

24. Yacobi NR, Phuleria HC, Demaio L, Liang CH, Peng CA, Sioutas C, et al. Nanoparticle effects on rat alveolar epithelial cell monolayer barrier properties. Toxicol In Vitro. 2007;21:1373-1381.

25. Hagens WI, Oomen AG, de Jong WH, Cassee FR, Sips AJAM. What do we (need to) know about the kinetic properties of nanoparticles in the body? Regul Toxicol Pharmacol. 2007;49:217-229.

26. Nel A, Xia T, Mädler L, Li N. Toxic Potential of Materials at the Nano level. Sci. 2006;311:622-627.

27. Donaldson K, Stone V, Duffin R, Clouter A, Schins R, Borm P. The quartz hazard: effects of surface and matrix on inflammogenic activity. J Environ Pathol Toxicol Oncol. 2001;20:109-118.

28. Baveye P, Laba M. Aggregation and toxicology of titanium dioxide nanoparticles. Environ Health Perspect. 2008;116:152-158.

29. Okuda-Shimazaki J, Takaku S, Kanehira K, Sonezaki S, Taniguchi A. Effects of titanium dioxide nanoparticle aggregate size on gene expression. Int J Mol Sci. 2010;11:2383-2392.

30. Li Y, Lee N, Song JS, Lee EG, Kim S. Synthesis and photocatalytic properties of nano bi-crystalline titania of anatase and brookite by hydrolizing $\mathrm{TiOCl}_{2}$ aqueous solution at low temeperatures. Res Chem Intermed. 2005;31:309-318.

31. Yamamoto A, Honma R, Sumita M, Hanawa T. Cytotoxicity evaluation of ceramic particles of different sizes and shapes. J Biomed Mat Res. 2004;68:244-256.

32. Kim JS. Antimicrobial effects of silver nanoparticles. Nanomed Nanotechnol Bio Med. 2007;3:95-101.
33. Shrivastava S, Bera T, Roy A, Singh G, Ramachandrarao P, Dash D. Characterization of enhanced antibacterial effects of novel silver nanoparticles. Nanotech. 2007:18:103-112.

34. Huang L, Dianqing L, Evans DG, Duan X. Influence of nano-Mgo particle size on bactericidal action against Bacillus subtilis var niger. Chinese Science Bulletin. 2005;50:514-519.

35. Fung MC, Bowen DL. Silver products for medical indications: riskbenefit assessment. Clin Toxicol. 1996;34:119-126. http://ehp03. niehs.nih.gov/article/findArticle.action?author=Fung\&title=Silver $\% 20$ products $\% 20$ for $\% 20$ medical $\% 20$ indications: $\% 20$ risk-benefit $\% 20$ assessment.\&source $=$ Clin\%20Toxicol\&year=1996\&firstpage $=119$.

36. Faunce TA. Safety of nanoparticles in sunscreens. MJA. 2009;190: 20-28.

37. Hallmans G, Liden S. Penetration of $65 \mathrm{Zn}$ through the skin of rats. Acta Dermatol Venereol. 1979;59:105-112.

38. Kapur SP, Bhussry BR, Rao S, Harmuth-Hoene E. Percutaneous uptake of zinc in rabbit skin. Proc Soc Exp Biol Med. 1974;145:932-399.

39. Rupesh KH, Bose S. Carbon nanotube based composites - a review. J Miner Mat Chara Eng. 2005;4:31-46.

40. Yadav BC, Kumar R. Structure, properties and applications of fullerenes. Inte J Nanotech Appl. 2008;2:15-24.

41. Wang C. C60 and Water-soluble fullerene derivatives as antioxidants against radical-initiated lipid peroxidation. J Med Chem. 1999;42: 4614-4620.

42. Petrus M, Suijkerbuijk W. Risk assessment of engineered carbonaceous nanoparticles in the aquatic environment. Masters Thesis. Toxicol Envir Health. November 2008. The Netherlands.

43. Kamat JP, Devasagayam TP, Priyadarsini KI, Mohan H, Mittal JP. Oxidative damage induced by the fullerene $\mathrm{C} 60$ on photosensitization in rat live rmicrosomes. Chem Biol Interact. 1998;114:145-159.

44. Levi N. $\mathrm{C}_{60}$-Fullerenes: detection of intracellular photoluminescence and lack of cytotoxic effects. J Nanobiotec. 2006;4:14-18.

45. Muller J, Huaux F, Moreau N. Respiratory toxicity of multi-wall carbon nanotubes. Toxicol Appl Pharmaco. 2005;207:221-231.

46. Donaldson K, Borm P. Particle and fiber toxicology, a new journal to meet a real need. Par Fibre Toxicol. 2004;1:1-3.

47. Willems I, Konya Z, Colomer J-F, et al. Control of the outer diameter of thin carbon nanotubes synthesized by catalytic decomposition of hydro-carbons. Chem Phys Lett. 2000;317:71-76.

48. Wing Lam C, James John T, McCluskey R, Hunter L. Pulmonary toxicity of single-wall carbon nanotubes in mice 7 and 90 days after intratracheal instillation. Toxicol Sci. 2004;77:126-134.

49. Ajayan PM, Charlier J, Rinzler AG. Carbonnanotubes: from macromolecules to nanotechnology. Proc Natl Acad Sci U S A. 199; 96:14199-14200.

50. Ajayan PM, Ebbesen TW. Nanometre-size tubes of carbon. Rep Prog Phys. 1997;60:1025-1062.

51. Bates DV, Fish BR, Hatch TF, Mercer TT, Morrow PE. Deposition and retention models for internal dosimetry of the human respi-ratory tract. Health Phys. 1967;12:173-207.

52. Azizian J, Tahermansouri H, Biazar E, Heidari S, Chobfrosh Khoei D. Functionalization of carboxylated multiwall nanotubes with imidazole derivatives and their toxicity investigations. Inter J Nanomed. 2010;5: 907-914.

53. Revell PA. The biological effects of nanoparticles. Nanotec Percep. 2006;2:283-298.

54. Burleson T, Yusuf N, Stanishevsky A. Surface modification of nanodiamonds for biomedical application and analysis by infrared spectroscopy. J Ach Mat Manufac Eng. 2009;37:568-574.

55. Bauer CE, Inspektor A, Oles EJ. A comparative machining study of diamond-coated tools made by plasma torch, microwave, and hot filament techniques. Acad Pro Eng Sci. 2003;28:933-944.

56. Kim Do-Kyung. Superparamagnetic Iron Oxide Nanoparticles for Biomedical Applications. Stockholm 2001 Licentiate Thesis. Royal Institute of Technology Department of Material Science and Engineering. 
57. Rishikesh MS. Nanosized cancer cell-targeted polymeric immunomicelles loaded with super paramagnetic iron oxide nanoparticles. Nanopart Res. 2009;6:1-9.

58. Mahmoudi M, Simchi A, Milani AS, Stroeve P. Cell toxicity of superparamagnetic iron oxide nanoparticles. J Colloid Inter Sci. 2009; 336(2):510-518.

59. Stroh A. In vivo detection limits of magnetically labeled embryonic stem cells in the rat brain using high-field (17.6 T) magnetic resonance imaging. Neuro Image. 2005;2:4635-4645.

60. Biazar E, Beitollahi A, Rezayat SM. Effect of the mechanical activation on size reduction of crystalline acetaminophen drug particles. Int $J$ Nanomed. 2009;4:283-287.

61. Majeti N, Kumar R. Nano and microparticles as controlled drug delivery devices. J Pharm Pharmaceut Sci. 2000;3:234-258.

62. Ochekpe Nelson A, Olorunfemi Patrick O, Nanotechnology and drug delivery part 1: background and applications. J Pharm Res. 2009;8: 265-274.

63. Ochekpe Nelson A, Olorunfemi Patrick O. Nanotechnology and drug delivery part 2: nanostructures for drug delivery. J of Pharm Res. 2009; 8:275-287.

64. Pinto RC. Nanoencapsulation I. Methods for preparation of drug-loaded polymeric nanoparticles. Nanomed: Nanotech Biol Med. 2006;2: 8-21.

65. Harivardhan RL, Murthy RSR. Phrmacokinetics and biodistribution studies of doxorubicine loaded poly(butyl cyanoacrylate) nanoparticles synthesized by two different techniques. Biomed. 2004;148: $161-166$.
66. Allemann E. Drug loaded nanoparticles preparation methods and drug targeting issues. Eur J Pharmbiopharm. 1993;39:173-191.

67. Stephen JD. Biodistribution of poly(butyl 2-cyanoacrylate) nanoparticles in rabbits. Int J Pharmaceutics. 1986;34:145-152.

68. Magenheim MY, Benita S. A new in vitro technique for the evaluation of drug release profile from colloidal carriers - ultrafiltration technique at low pressure B. Int J Pharmaceutics. 1993;94:115-123.

69. Corrigan OI, Li X. Quantifying drug release from PLGA nanoparticulates. Euro J Pharm Sci. 2009;37:477-485.

70. Silva GA. Nanotechnology approaches for drug and small molecule delivery across the blood brain barrier. Sur Neuro. 2007;67:113-116.

71. Esmaeil Biazar, Mahdi Rezayat S, Naser Montazeri. The effect of acetaminophen nanoparticles on liver toxicity in a rat model. Int $J$ Nanomed. 2010;5:197-201.

72. Ndungu JM. Targeted delivery of paclitaxel to tumor cells: synthesis and in vitro evaluation. J Med Chem. 2010;53:3127-3132.

73. Singh SS. Nanotechnology-based drug delivery systems. J Occup Med Toxicol. 2007;2:16-24.

74. Robe A. Quantum dots in axillary lymph node mapping: biodistribution study in healthy mice. BMC Cancer. 2008;8:111-116.

75. Oberdorster G, Stone V, Donaldson K. Nanotoxicology Toxicology of nanoparticles: a historical perspective. Nanotoxicol. 2007;1:2-25.

76. Sadauskas E. Biodistribution of gold nanoparticles in mouse lung following intratracheal instillation. Chem Cen J. 2009;3:16-21.
International Journal of Nanomedicine

\section{Publish your work in this journal}

The International Journal of Nanomedicine is an international, peerreviewed journal focusing on the application of nanotechnology in diagnostics, therapeutics, and drug delivery systems throughout the biomedical field. This journal is indexed on PubMed Central, MedLine, CAS, SciSearch $®$, Current Contents $₫ /$ Clinical Medicine,

\section{Dovepress}

Journal Citation Reports/Science Edition, EMBase, Scopus and the Elsevier Bibliographic databases. The manuscript management system is completely online and includes a very quick and fair peer-review system, which is all easy to use. Visit http://www.dovepress.com/ testimonials.php to read real quotes from published authors 